\title{
CELL-SEEDED POLYURETHANE-FIBRIN STRUCTURES - A POSSIBLE SYSTEM FOR INTERVERTEBRAL DISC REGENERATION
}

\author{
C. Mauth ${ }^{1 \S}$, E. Bono ${ }^{1 \S}$, S. Haas ${ }^{1}$, G. Paesold ${ }^{2}$, H. Wiese ${ }^{3}$, G. Maier ${ }^{3}$, N. Boos ${ }^{2}$ and U. Graf-Hausner ${ }^{1 *}$ \\ ${ }^{1}$ School of Life Sciences and Facility Management, Institute of Chemistry and Biological Chemistry, Campus \\ Reidbach, Einsiedlerstrasse 31, CH-8820 Waedenswil/Zurich, Switzerland \\ ${ }^{2}$ University Hospital Balgrist, Department of Orthopedics, Forchstrasse 340, CH-8008 Zurich, Switzerland \\ ${ }^{3}$ polyMaterials AG, Innovapark 20, D-87600 Kaufbeuren, Germany
}

$\S$ Both authors contributed equally

\begin{abstract}
Nowadays, intervertebral disc (IVD) degeneration is one of the principal causes of low back pain involving high expense within the health care system. The long-term goal is the development of a medical treatment modality focused on a more biological regeneration of the inner nucleus pulposus (NP). Hence, interest in the endoscopic implantation of an injectable material took center stage in the recent past. We report on the development of a novel polyurethane (PU) scaffold as a mechanically stable carrier system for the reimplantation of expanded autologous IVDderived cells (disc cells) to stimulate regenerative processes and restore the chondrocyte-like tissue within the NP. Primary human disc cells were seeded into newly developed PU spheroids which were subsequently encapsulated in fibrin hydrogel. The study aims to analyze adhesion properties, proliferation capacity and phenotypic characterization of these cells. Polymerase chain reaction was carried out to detect the expression of genes specifically expressed by native IVD cells. Biochemical analyses showed an increased DNA content, and a progressive enhancement of total collagen and glycosaminoglycans (GAG) was observed during cell culture. The results suggest the synthesis of an appropriate extracellular matrix as well as a stable mRNA expression of chondrogenic and/or NP specific markers. In conclusion, the data presented indicate an alternative medical approach to current treatment options of degenerated IVD tissue.
\end{abstract}

Keywords: intervertebral disc, polyurethane, fibrin, scaffold, nucleus pulposus, cell encapsulation.

* Address for correspondence:

Ursula Graf-Hausner

Campus Reidbach, Einsiedlerstrasse 31

CH-8820 Waedenswil/Switzerland

Telephone Number: +41589345518

FAX Number: +41 589345659

E-mail: ursula.graf@zhaw.ch

\section{Introduction}

Intervertebral disc (IVD) degeneration is an age-related process affecting the biomechanical properties of the spine and is assumed to be one of the principal causes of lower back pain. During disc degeneration, dehydration of the nucleus pulposus (NP), due to loss of proteoglycans and disorganization of the extracellular matrix causes an inefficient transfer of load between the vertebral bodies leading to further degeneration or even to damage of the annulus fibrosus (AF) (disc herniation) (Urban and Roberts, 2003).

Current treatments are highly invasive surgical procedures such as spinal fusion and discectomy. The clinical solution is either complete disc removal in conjunction with spinal interbody fusion or total disc arthroplasty (Mayer, 2005). Both approaches are not biological nor do they preserve the natural disc function, and revision surgery is frequently necessary. In contrast, biological approaches aim at the stimulation of disc regeneration (Paesold et al., 2007) to avoid or at least delay surgical procedures.

A potential alternative could be a tissue engineering approach to regenerate the inner NP tissue at an earlystage of the degeneration process. The focus should be on biocompatible materials that allow three-dimensional (3D) cell culture and provide initial mechanical stability after implantation. Thus, cells are given time to synthesize the extracellular matrix required to resist the compressive loads caused by daily movements. Moreover, the cellular scaffold has to promote cell adhesion and integration, and needs to allow appropriate transport of nutrients and waste products through the structure. For clinical application it is crucial that the material can be implanted by minimally invasive surgery, while cells maintain their viability and are homogenously distributed.

Different synthetic biomaterials have been studied to generate in vitro tissue constructs for subsequent disc augmentation, and are at different levels of preclinical and clinical investigations (Di Martino et al., 2005). Studies have been performed using macroporous scaffolds such as collagen, hyaluronan, calcium phosphates, polylactide (Yang et al., 2005; Revell et al., 2007; Séguin et al., 2004; Brown et al., 2005) as well as hydrogels like fibrin, gelatin, or chitosan (Yang et al., 2005; Bertram et al., 2005; Roughley et al., 2006; Yang et al., 2008). In the present study the fibrin glue Tissucol was chosen, focusing the attention on developing a clinical applicable product whereby the fibrin is already approved by the Federal Drug 
Agency (FDA) and applied in surgery. Fibrin is a biocompatible, microporous structure which enables cell migration as well as diffusion of cell signaling factors and waste products. The hydrogel is injectable and polymerizes in vivo at body temperature due to a combination of fibrinogen and thrombin components. Despite these advantages, fibrin gel lacks biomechanical resistance and chemical stability concerning spinal load. As demonstrated in various studies in cartilage regeneration (Peretti et al., 2000; Caterson et al., 2001; Lee et al., 2005; Eyrich et al., 2007a), fibrin needs to be combined with a more stable material. Here, we combined fibrin hydrogel with polyurethane (PU) in spherical form to provide a suitable structure for isolated human disc cells.

The PU we used provides a macroporous, biocompatible and biodegradable system with sufficient elasticity, and stiffness to resist compressive loading while maintaining structural integrity (Eyrich et al., 2007b). Indeed, PU has been used as a cancellous bone graft substitute in animals and as a scaffold in cartilage tissue engineering (Lee et al., 2005; Gogolewski et al., 2006; Gogolewski et al., 2007; Liu et al., 2004; Grad et al., 2006; Guelcher et al., 2006). Yang et al. (2009) selected PU material as a scaffold to regenerate the annulus fibrosus, demonstrating that the modulation of the PU surface chemistry can influence cell adhesion and early tissue formation. Thus, the PU foam provides an initial cell carrier matrix to resist the mechanical load immediately after implantation, while fibrin acts as a space-filling compound and 3D structure to retain cells at the injection site and maintain their characteristic phenotype.

Hence the study presents the assessment of the applicability of the PU-fibrin structure designed for intervertebral disc augmentation. Therefore, human disc cells were monolayer-expanded in vitro and cultured in two different 3D culture systems, the PU-fibrin structure and a pellet culture system. Cell adhesion, proliferation capacity as well as phenotypic cell characterization were carefully analyzed.

\section{Materials and Methods}

\section{Cell isolation and expansion}

Intervertebral disc tissue was obtained during discectomy of human donors at the University Hospital Balgrist. The approval of the local ethical committee was obtained for the use of the samples. The biopsy was transported on ice in Dulbecco's Modified Eagle Medium (DMEM) containing F12 and 1\% antibiotic-antimicotic solution (AAS) (InVitrogen Gibco, Basel, Switzerland). The biopsy was washed twice in sterile phosphate-buffered saline (PBS) immediately before being minced into pieces. Tissue samples were weighed and digested at $37^{\circ} \mathrm{C}$ for 16 hours with $0.025 \% \quad(w / v)$ NB4 collagenase (Serva Electrophoresis, Heidelberg, Germany) in DMEM/F12 containing 1\% AAS. Afterwards cell suspension was filtered through a $70 \mathrm{~mm}$ cell strainer (Falcon, Becton Dickinson, Allschwil, Switzerland) and centrifuged at 200xg for 10 minutes. Cell viability was assessed by the trypan blue dye-exclusion assay. Human disc cells $\left(2 \times 10^{3}\right.$ cells $/ \mathrm{cm}^{2}$ ) were then expanded in monolayer culture using DMEM/F12 supplemented with $5 \%$ fetal bovine serum (FBS) (Gibco), 1\% penicillin/streptomycin solution (PSS) (Sigma-Aldrich, Basel Switzerland), $5 \mathrm{ng} / \mathrm{mL}$ basic fibroblast growth factor (bFGF) (Invitrogen) and $1 \mathrm{ng} / \mathrm{mL}$ transforming growth factor (TGF)-b1 (Lubioscience, Lucerne, Switzerland), and cultured at $37^{\circ} \mathrm{C}$ and $5 \% \mathrm{CO}_{2}$ for about 14 days until reaching confluence. The medium was changed every second to third day.

\section{Pellet culture}

The pellet system was selected as positive control in the gene expression studies, which were performed in order to confirm the redifferentiation of disc cells after the monolayer cultivation. Therefore, disc cells were harvested after proliferation and pellet formation was induced as follows: $5 \times 10^{5}$ cells were suspended in $3 \mathrm{~mL}$ of differentiation medium containing DMEM high glucose (Gibco), 1\% PSS, 1\% solution containing insulin, human transferrin, and selenous acid (ITS) (BD Biosciences, Allschwil, Switzerland), 150 $\mathrm{MM}$ ascorbic acid-2 phosphate (ASC) (Sigma), 100nM dexamethasone (Dex) (Sigma), $1 \mathrm{mM}$ sodium pyruvate (Sigma) and $10 \mathrm{ng} / \mathrm{mL}$ TGF-b1. The cell suspension was gently centrifuged at $200 \mathrm{xg}$ for 3 minutes in polystyrene tubes to form pellets. Cell culture was performed over 14 days and the medium was changed every second day.

\section{Polyurethane scaffolds}

PU scaffolds were synthesized and kindly provided by polyMaterials AG (Kaufbeuren) (Wiese and Maier, 2006). Porous and interconnective foamy PU structures with a hydrophobic surface were prepared as follows: $3 \mathrm{~g}$ of poly(caprolactone) diol ( $\mathrm{Mn}=2000 \mathrm{~g} / \mathrm{mol}$, Acros Organics, Belgium) and $3 \mathrm{~g}$ of poly(caprolactone) triol (crosslinker, $\mathrm{Mn}=900 \mathrm{~g} / \mathrm{mol}$, Acros Organics) were melted and mixed at $40^{\circ} \mathrm{C} .100 \mathrm{mg}$ of mannitol (Acros Organics) as nucleating agent, $1 \mathrm{~g}$ of methylal as blowing agent (Sigma), and $1.5 \mathrm{~g}$ isophorone diisocyanate (Sigma) were added and mixed. The reaction was started by adding $50 \mathrm{~mL}$ of 1.8 diazabicyclo[5.4.0] undec-7-en (Acros Organics) and mixing vigorously. The foam was cured at $65^{\circ} \mathrm{C}$ after one hour. The isophorone diisocyanate was added in a slight excess because of side reactions (e.g. allophanate reaction) increasing the crosslinking degree and leading to a higher stiffness of the polymer. The PU foam had a porosity of $80 \%$ with pore size of 80 to $300 \mathrm{~mm}$ and high interconnectivity.

For cell culture experiments the PU foam structure was minced into pieces using a Dispomix ${ }^{\circledR}$ (Axon Lab AG, Baden-Dettwill, Switzerland) tabletop homogenizer with a predefined profile ( 20 seconds at the rotational speed of $4000 \mathrm{rpm}$ and 5 seconds at $4000 \mathrm{rpm}$ in the opposite direction). The obtained spheroids were observed under the stereomicroscope showing an average particle size of $1 \pm 0.4 \mathrm{~mm}$ in diameter. PU scaffolds were vacuum-dried and distributed in 96-well plates (5mg PU/well $=88.21 \pm$ 26.05 PU spheroids/well). The PU spheroids were then sterilized in $75 \%$ ethanol for 60 minutes followed by three wash steps in sterile bidistilled water. 


\section{Fibrin hydrogel}

TISSUCOL Duo S immuno kit (Baxter Biosurgery, Volketswil, Switzerland) was used to prepare the fibrin hydrogel which served as connective material between PU foam spheroids and provided a biocompatible, injectable extracellular matrix. The product components were mixed as described in the manufacturer's instructions leading to a final concentration of $70-110 \mathrm{mg} / \mathrm{mL}$ fibrinogen, $500 \mathrm{IU} /$ $\mathrm{mL}$ thrombin (human), and $3000 \mathrm{KIU} / \mathrm{mL}$ aprotinin (bovine).

\section{Cell seeding and preparation of PU-fibrin structure}

After proliferation in monolayer culture, $1.2 \times 10^{5}$ disc cells were suspended in $100 \mu \mathrm{l}$ of proliferation medium (DMEM/ F12 supplemented with $5 \%$ FBS, $1 \%$ PSS, $5 \mathrm{ng} / \mathrm{mL}$ FGF, $1 \mathrm{ng} / \mathrm{mL}$ TGF- $\beta 1$ and $130 \mu \mathrm{M}$ ASC), and dynamically seeded directly onto $5 \mathrm{mg}$ PU spheroids per well, which had been pre-incubated over night with 5\% serum containing medium to improve cellular adhesion. In particular, cells were seeded onto PU particles in suspension and incubated for 30 minutes. Subsequently, the cell suspension was mixed to avoid cell sedimentation and stimulate the adhesion onto PU spheroids. This procedure was repeated one more time before adding fresh proliferation medium $(150 \mu \mathrm{l} /$ well). In order to measure the efficiency of the dynamic seeding procedure, PU samples were transferred into new wells, and the remaining non-adhered cells were counted. These seeded PU spheroids were pre-cultured for 3 days in proliferation medium and then embedded in fibrin hydrogel $(130 \mu \mathrm{l}$ fibrin/5mg PU). The samples were incubated for one hour at $37^{\circ} \mathrm{C}$ to permit gelation. Afterwards the structures (height $6.5-7 \mathrm{~mm} \times$ diameter $8.6 \mathrm{~mm}$ ) were transferred to 24 well plates and covered with $1.2 \mathrm{~mL}$ differentiation medium. The day of fibrin supplementation to PU spheroids was defined as day 0 of analysis. The structures were cultured for 3, 7 and 14 days, and medium was changed every second day.

\section{Histological analysis}

In order to visualize cell adhesion on PU itself, spheroids seeded with disc cells were analyzed at day 0 and 14 of culture. Samples were gently washed with PBS, fixed in $3.7 \%$ formaldehyde and washed again with PBS before staining with 4',6-diamidino-2-phenylindole (DAPI) (1 $\mu \mathrm{g} /$ $\mathrm{mL})$. The stained PU spheroids were analyzed using fluorescence microscopy (Axioskop 2 plus microscope equipped with the Axio Cam color camera, Zeiss, Göttingen, Germany).

Moreover, cells were mixed directly into fibrin and, cultivated under the same conditions as PU-fibrin structures. The cell distribution was analyzed by DAPI staining. In particular cell containing fibrin gels (day 0 and 14) as well as PU-fibrin composites (day 3, 7 and 14) were fixed in $3.7 \%$ formaldehyde and gently rinsed three times with PBS. The structures were then shock frozen in liquid nitrogen and $20 \mu \mathrm{m}$ thin sections were prepared by sectioning in the Cryostat HM 550 OMP (Thermo Scientific, Waltham, MA, USA). Nuclei were stained with DAPI as previously described. The samples were observed using light and fluorescence microscopy.

\section{Biochemical analysis}

On days 3, 7 and 14 of culture PU-fibrin structures were digested in papain (Sigma) solution $(28 \mathrm{mg} / \mathrm{mL})$ for 6 hours at $58^{\circ} \mathrm{C}$. Total DNA content was determined by the Hoechst 33258 dye (Roche, Basel Switzerland) assay on an aliquot of the digested sample using a plate reader (UV-visible spectrophotometer, Cary 50 Scan; Varian, Palo Alto, CA, USA) at $355 \mathrm{~nm}$ excitation wavelength and $460 \mathrm{~nm}$ emission wavelength. The DNA content of each sample was quantified by interpolating values from a linear standard curve generated from calf thymus DNA.

Total sulfated glycosaminoglycan (GAG) content as well as the collagen amount was assessed in the same digestion supernatant. GAG amount was measured in a dimethylmethylene blue (Fluka; Sigma) buffer solution (pH 3.0). Absorbance was measured at $630 \mathrm{~nm}$ and a standard curve was generated using chondroitin-4-sulfate (Barbosa et al., 2003). The GAG to DNA ratio was determined by dividing GAG $(\mu \mathrm{g})$ by DNA $(\mu \mathrm{g})$ per each digested PU-fibrin structure.

Collagen content was detected using the chloramineT staining method. Briefly, samples were hydrolyzed with $10 \mathrm{M} \mathrm{NaOH}$, neutralized and stained with chloramine-T solution (Sigma). Absorption was measured at 540nm. The total collagen content was quantified by interpolating values $(\mu \mathrm{g})$ from a linear standard of gelatin-hydrolyzate (Sigma) (Hoemann et al., 2002). The collagen to DNA ratio was determined by dividing collagen $(\mu \mathrm{g})$ by DNA $(\mu \mathrm{g})$ per each digested sample.

\section{Gene expression analysis}

Phenotypic characterization was performed with fresh biopsy (100mg), monolayer cultures of isolated human disc cells ( $10^{6}$ cells) (after 10 days), and also with pellet cultures (after 14 days) and PU-fibrin composites (after 14 days). Cells were lysed in TRIzol reagent (Invitrogen) and total cellular RNA was isolated in accordance with the recommendations of the manufacturer. For biopsy samples and the PU-fibrin composites, the samples were furthermore mechanically homogenized in Trizol using the Dispomix $^{\circledR}$. The purified samples were extracted by phenol/chloroform, precipitated with isopropanol, washed with $70 \%$ ethanol and resuspended in RNase-free water. RNA concentrations were assessed spectrophotometrically (UV-visible spectrophotometer, Cary 50 Scan, Varian) at $260 \mathrm{~nm}$. 400ng of total RNA was reverse transcribed (Superscript $^{\mathrm{TM}}$ II, Invitrogen) and analyzed by Reverse Transcriptase-PCR (RT-PCR) (Hotstar Taq DNA polymerase; Qiagen, Hilden, Germany) using genespecific primers (Table 1) for collagen $1(\mathrm{Col} 1 \alpha 1)$, collagen II (Col2 $\alpha 1)$, aggrecan and transcription factor Sox9 (Lin et al., 2005), hypoxia inducible factor-1 (HIF) $1 \alpha$ isoform (Gao et al., 2005), metalloproteinase-2 (MMP2) (Shan et al., 2005), glycosylphosphatidylinositol-anchored cell surface protein CD24 (Fujita et al., 2005), glucose transporter (GLUT-1) (Robey et al., 2005), and glyceraldehyde 3-phosphate dehydrogenase (GAPDH) as a housekeeping gene (Sanchez et al., 2005). The PCR fragments were resolved on a $1.5 \%$ agarose gel and stained with ethidium bromide. 

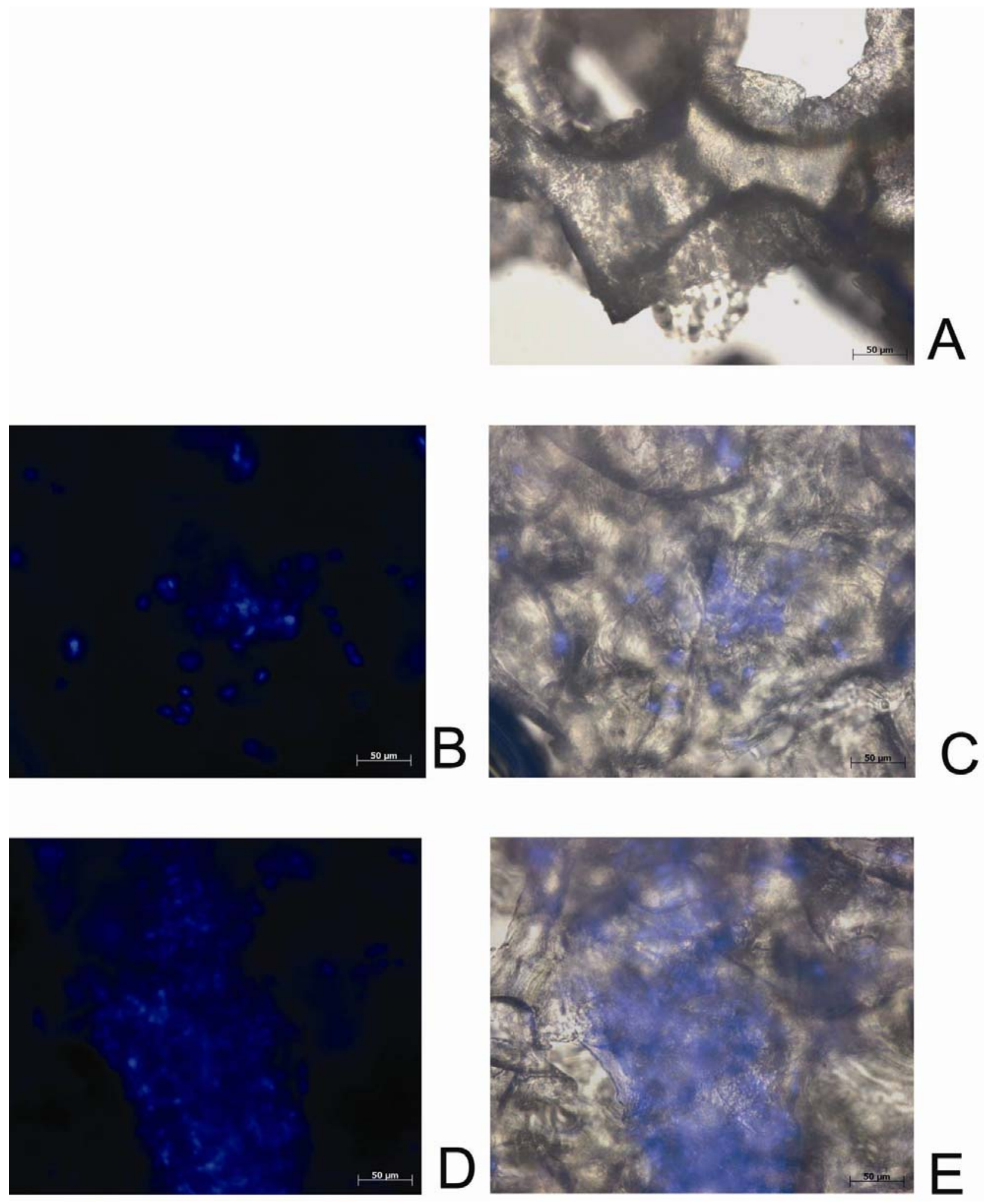

Figure 1. Human nucleus pulposus-like cells were seeded onto PU spheroids. The cell adhesion has been proven by DAPI staining. (A) PU spheroids without cells (blank). (B) Nuclei staining of adhered cells onto the PU material pre-cultured for 3 days with proliferation medium (day 0), observed by fluorescence microscopy, and (C) overlay with light microscopy. (D) Nuclei staining of disc-like cells after 14 days of culture with differentiation medium observed by fluorescence microscopy, and (E) overlay with light microscopy. 

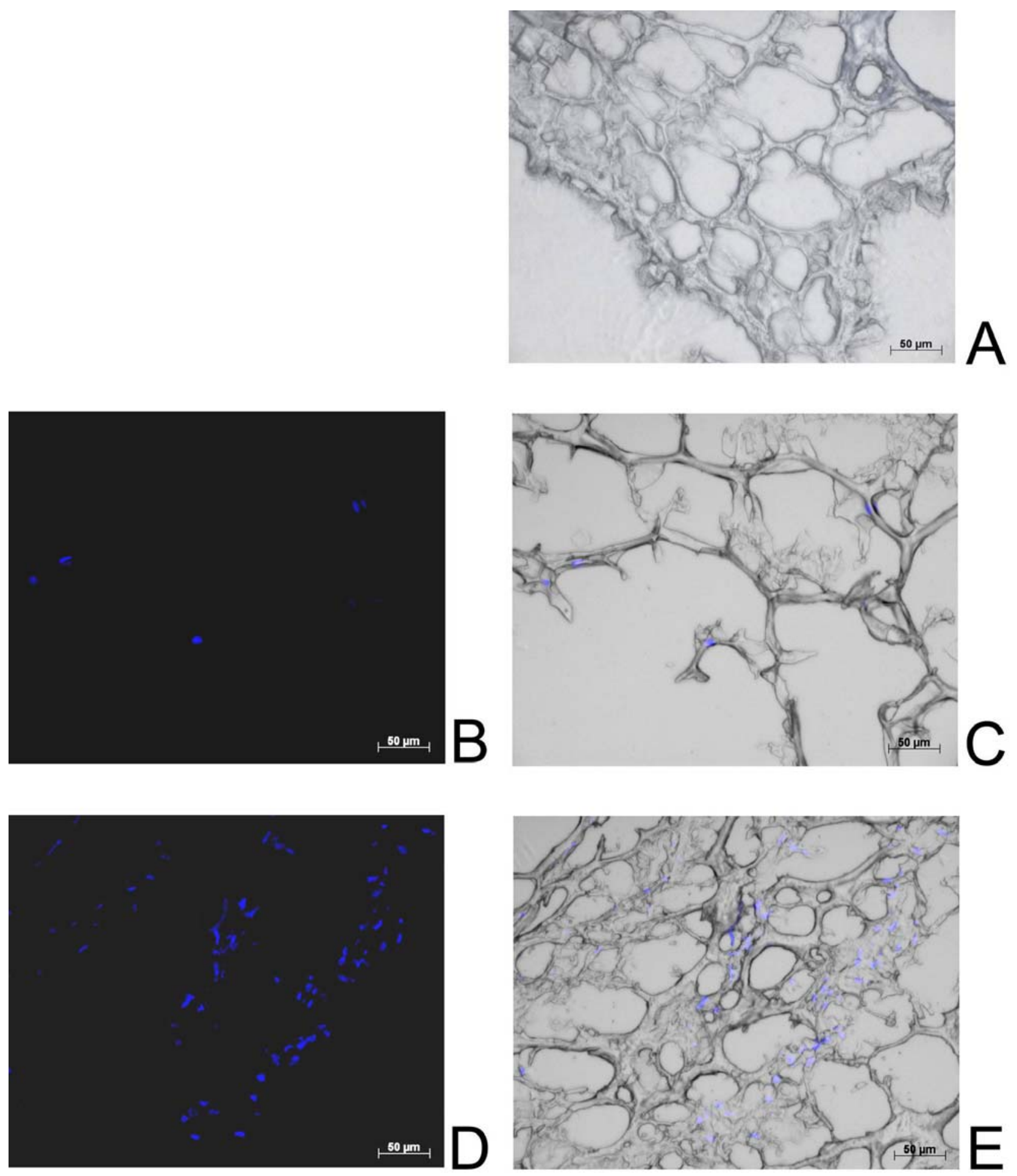

Figure 2. Human nucleus pulposus-like cells were seeded in fibrin structures by adding them to the fibrin before its polymerization. (A) Fibrin structure without cells (blank). (B) Nuclei staining of adhered cells in the matrix, pre-cultured for 3 days with proliferation medium (day 0), observed by fluorescence microscopy and (C) overlay with light microscopy. (D) Nuclei staining of disc-like cells after 14 days of culture with differentiation medium observed by fluorescence microscopy, and (E) overlay with light microscopy. 


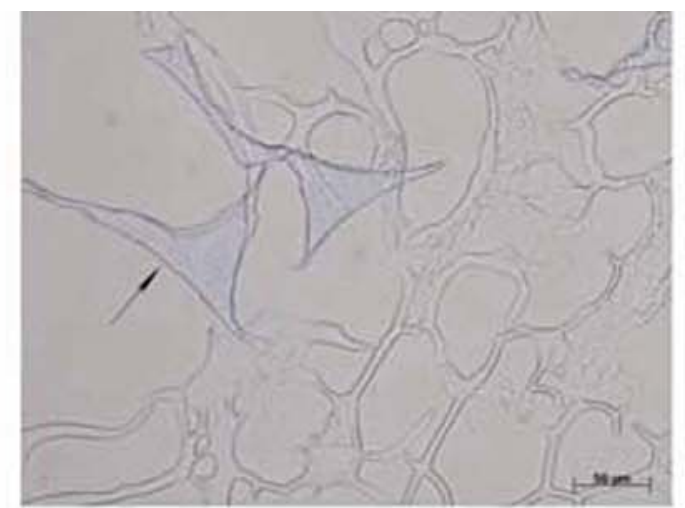

A
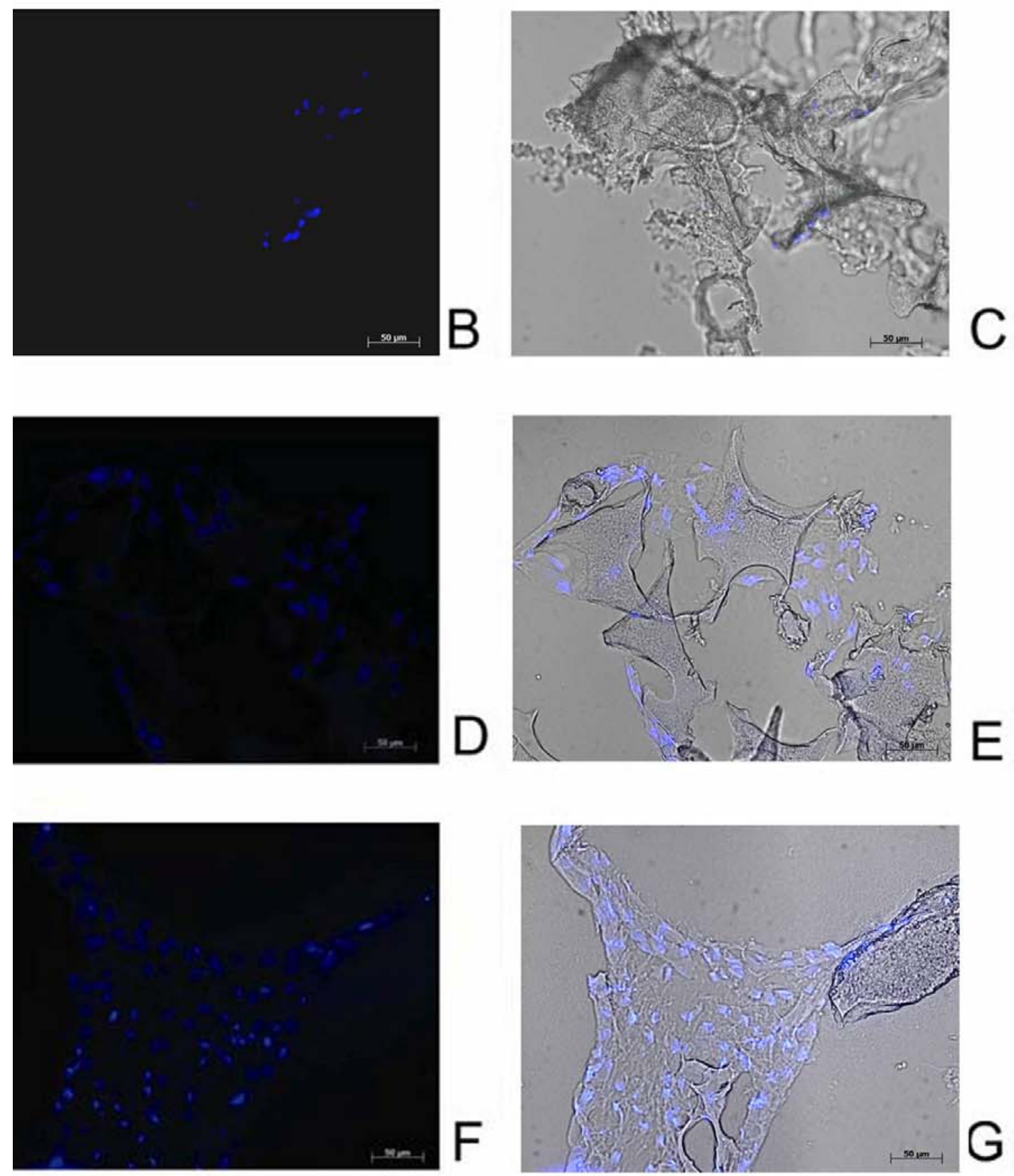

Figure 3. DAPI stained cryosections of PU spheroids seeded with human disc-like cells and embedded into fibrin hydrogel. (A) PU spheroids embedded in fibrin hydrogel without cells (blank). Cell expansion and distribution into the PU-fibrin structure after (B) 3 days, (D) 7 days and (F) 14 days observed by fluorescence microscopy, and $(\mathbf{C}, \mathbf{E}, \mathbf{G})$ overlay with light microscopy. The black arrows indicate the PU spheroids. 

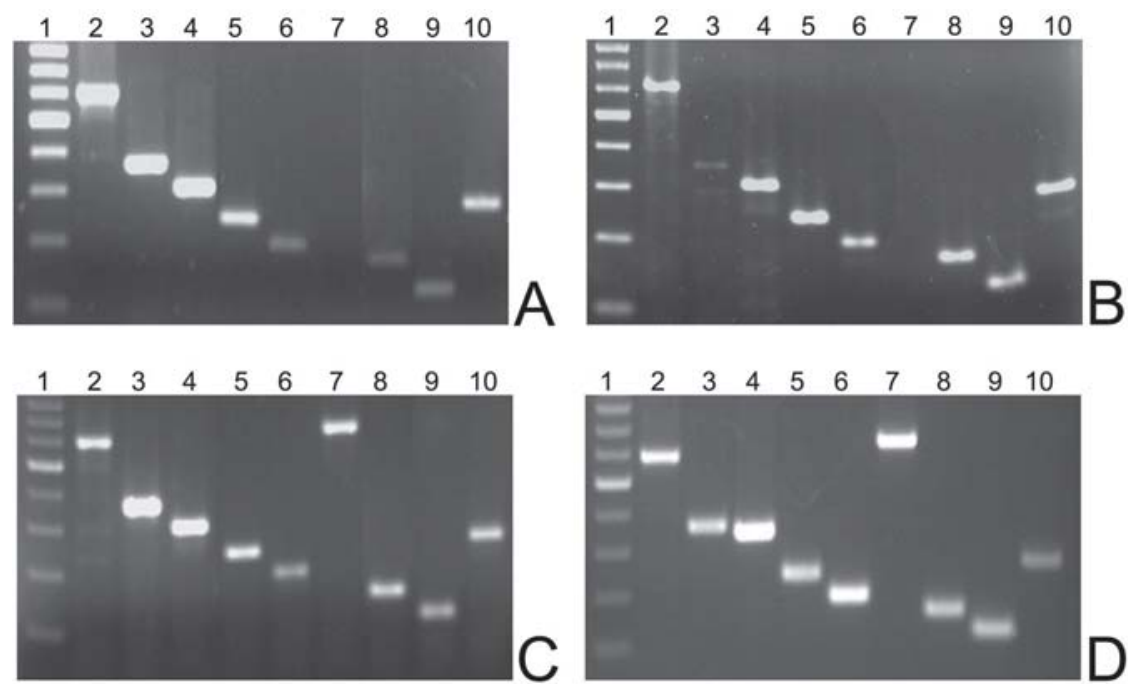

Figure 4. Gene expression analyses of one culture sample as example. Total RNA was isolated from (A) disc biopsy, (B) monolayer culture after 10 days, (C) pellet culture, and (D) PUfibrin structure after 14 days in culture. The RNA was subjected to reverse transcriptase-PCR reaction and PCR products were resolved on a $1.5 \%$ agarose gel. DNA ladder of 100bp (lane 1), expression of collagen type I (lane 2 ) and type II (lane 3), aggrecan (lane 4), HIF-1 alpha (lane 5), MMP2 (lane 6), SOX9 (lane 7), GLUT-1 (lane 8), CD24 (lane 9) and GAPDH (lane 10).

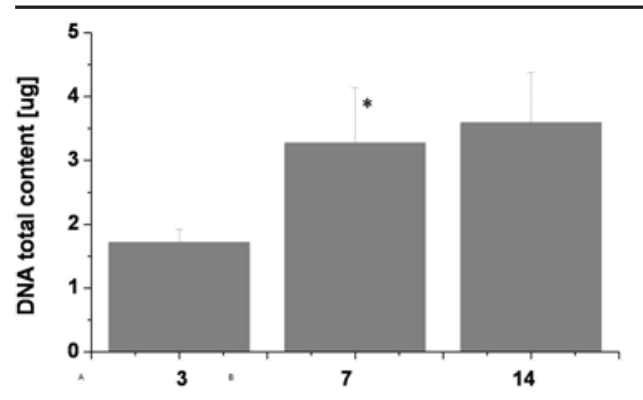

A

Cultivation time [d]

B

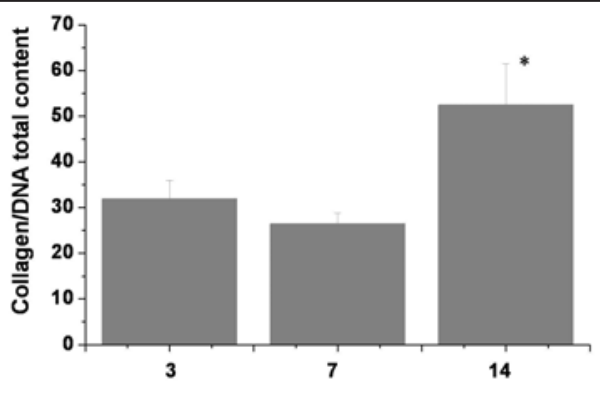

Cultivation time [d]

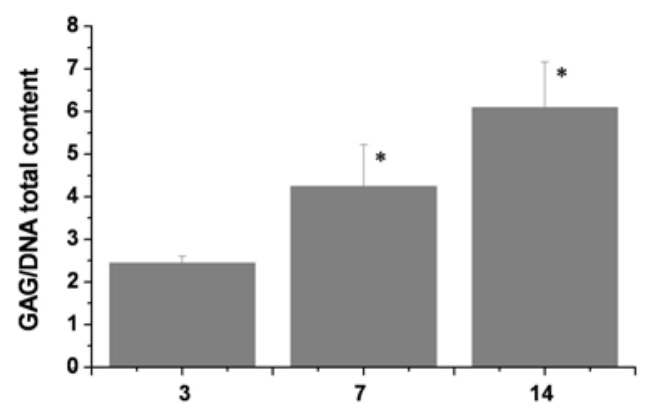

C
Figure 5 Biochemical analyses of (A) DNA content, (B) collagen and (C) GAG to DNA ratio within PU-fibrin structures after 3, 7 and 14 days of culture. The collagen/DNA ratio as well as the GAG/DNA ratio was determined by dividing the extracellular matrix component $(\mu \mathrm{g})$ by DNA $(\mu \mathrm{g})$ per each PU-fibrin structure. ${ }^{*} p<0.05$ compared cultures of 7 versus 3 days, and 14 versus 7 days in culture, respectively.

\section{Statistical analysis}

The statistical significance of total DNA, GAG and collagen content of PU-fibrin structures after 3, 7 and 14 days in culture were analyzed by ANOVA and the post hoc Fisher's PLSD (Protected Least Significant Difference) test. A $p$ value $<0.05$ was considered to be statistically significant

\section{Results}

\section{Cell culture and scaffold characterization}

For our experiments 18 biopsies were processed obtaining an average yield of $2.4 \pm 0.6 \times 10^{5}$ cells per g wet weight. The mean age of the donors was $43.6 \pm 17.4(57 \%<40$ and $43 \%>40$ years age; $27 \%$ men and $73 \%$ women). 68 $\pm 5 \%$ of isolated cells were found to adhere to the polystyrene surface of culture dishes and reached $80 \%$ confluence after 10 days. NP and AF cells were maintained in co-culture since cross-contamination of NP cells with AF cells was not avoidable during the discectomy. Thus, the duration of isolation procedure was minimized, and closely reflected the procedure that would be used during the targeted clinical application.

The cell adhesion efficiency onto hydrophobic PU spheroids was detected 2 hours after seeding. Cell seeded PU spheroids were transferred to new wells for further cultivation. The remaining non-adhered cells were counted and subtracted from the total cell seeding concentration resulting in an adhesion efficiency of $78 \pm 9.6 \%$. Moreover, the presence and distribution of cells onto pre-incubated PU spheroids was analyzed 3 days after the seeding (day 0 ) and at day 14 using DAPI staining. Samples were analyzed by fluorescence microscopy (Fig. 1B,D), and overlaid light microscopy (Fig. 1C,E). Cell distribution was also investigated in pure fibrin gels at day 0 and 14 demonstrating their homogeneity within the matrix (Fig. 2B-E). 
Table1. Gene specific primers used for PCR amplification

\begin{tabular}{|c|c|c|c|c|}
\hline Gene & GenBank No & Primer sequence $\left(5^{\prime}-3^{\prime}\right)$ & Length & $\mathbf{T}_{\mathrm{m}}$ \\
\hline Col I (A1) & NM_000088 & $\begin{array}{l}\text { Fwd: CAT CTC CCC TTC GTT TTT GA } \\
\text { Rev: CTG TGG AGG AGG GTT TCA GA }\end{array}$ & $598 \mathrm{bp}$ & 51.0 \\
\hline Col II (A1) & $\begin{array}{l}\text { NM_033150 } \\
\text { NM_001844 }\end{array}$ & $\begin{array}{l}\text { Fwd: AGC ATT GCC TAT CTG GAC GAA G } \\
\text { Rev: AGA GTC CTA GAG TGA CTG AG }\end{array}$ & $361 \mathrm{bp}$ & 57.0 \\
\hline Aggrecan & $\begin{array}{l}\text { NM_013227 } \\
\text { NM_001135 }\end{array}$ & $\begin{array}{l}\text { Fwd: GAG GAG GGC TGG AAC AAG TAC C } \\
\text { Rev: GGT AAT TGC AGG GAA CAT CAT T }\end{array}$ & $343 \mathrm{bp}$ & 60.0 \\
\hline HIF-1 $\alpha$ & $\begin{array}{l}\text { NM_001530 } \\
\text { NM_181054 }\end{array}$ & $\begin{array}{l}\text { Fwd: AAT GCC ACC ACT ACC ACT GC } \\
\text { Rev: TAA CAC GTT AGG GCT TCT TGG A }\end{array}$ & $237 \mathrm{bp}$ & 53.0 \\
\hline MMP-2 & NM_004530 & $\begin{array}{l}\text { Fwd: AGT CTG AAG AGC GTG AAG TTT G } \\
\text { Rev: CCA GGT AGG AGT GAG AAT GC }\end{array}$ & $192 \mathrm{bp}$ & 52.0 \\
\hline SOX9 & NM_000346 & $\begin{array}{l}\text { Fwd: GAA CGC ACA TCA AGA CGG AG } \\
\text { Rev: TCT CGT TGA TTT CGC TGC TC }\end{array}$ & $631 \mathrm{bp}$ & 60.0 \\
\hline GLUT-1 & NM_006516 & $\begin{array}{l}\text { Fwd: TCA ATG CTG ATG ATG AAC CTG CT } \\
\text { Rev: GGT GAC ACT TCA CCC ACA TAC A }\end{array}$ & $164 \mathrm{bp}$ & 53.0 \\
\hline CD24 & NM_013230 & $\begin{array}{l}\text { Fwd: GCA CTG CTC CTA CCC ACG CAG ATT T } \\
\text { Rev: GCC TTG GTG GTG GCA TTA GTT GGA T }\end{array}$ & $122 \mathrm{bp}$ & 59.0 \\
\hline GAPDH & NM_002046 & $\begin{array}{l}\text { Fwd: TTG GTA TCG TGG AAG GAC TCA } \\
\text { Rev: TGT CAT CAT ATT TGG CAG GTT T }\end{array}$ & $270 \mathrm{bp}$ & 50.0 \\
\hline
\end{tabular}

Three days after cell seeding, PU spheroids were encapsulated into fibrin (Fig. 3), and cultured for 3, 7 or 14 days. During culture cells proliferated inside the PUfibrin structure as demonstrated by DAPI staining of cryosections (Fig. 3B-G). Moreover, the PU-fibrin structure remained transparent and stable after 14 days. Interestingly, while cells were attached to the PU surface on day 3 (Fig. 3B,C), after 7 days of culture the majority of the encapsulated cells had become detached from the PU and distributed into the fibrin structure (Fig. 3D,E). On day 14, the fibrin matrix was completely penetrated by cells, resulting in a uniform distribution (Fig. 3F,G). From day 14 onwards, the role of the PU spheroids in the composites appeared to be mainly to stabilize the structure of the $3 \mathrm{D}$ culture enhancing the elasticity and the rigidity of the hydrogel.

\section{Specific gene expression levels of 3D cultured disc} cells

Semi-quantitative PCR was used to investigate the cell phenotype by analyzing the expression of chondrogenic (collagen type I and II, aggrecan, and Sox9) and also NP cell specific mRNA levels (HIF, GLUT-1, MMP-2 and CD24). Phenotypic profiles were generated either from disc tissue, monolayer culture, or pellet cultures versus PU-fibrin structures (3D), an example being shown in Figure 4.

The biopsy analysis (Fig. 4A) showed similar expression of Col1 $\alpha 1$, Col $2 \alpha 1$ and aggrecan. Expression of Sox-9 could not be displayed. NP markers on the protein level as MMP-2, GLUT-1, and CD24 showed equal yet low expression levels, while HIF expression could be demonstrated. 
After expansion of isolated disc cells in monolayer culture (Fig. 4B), the expression of Coll $\alpha 1$ was observed. In comparison, Col $2 \alpha 1$ was found to be notably decreased when compared to the respective mRNA levels of biopsy material. HIF, GLUT-1, MMP-2 and CD24 were expressed, while Sox9 was not detected. Expression levels were further analyzed in pellet cultures and compared to cultures in PU-fibrin structures. In pellet culture (Fig. 4C) a predominant upregulation of Col $2 \alpha 1$ versus Col1 $\alpha 1$ was observed, and also aggrecan was highly expressed. HIF, MMP, GLUT-1 and CD24 showed no notable changes compared to monolayer culture. However, Sox 9 mRNA expression was present.

Disc cells encapsulated into PU-fibrin structures also presented a high expression of extracellular matrix specific mRNA levels such as Col1 $\alpha 1$ and $\mathrm{Col} 2 \alpha 1$, and aggrecan. HIF, GLUT-1, CD24 and MMP-2 mRNA expression showed no striking changes compared to pellet cultures. The expression of Sox 9 was the most noticeable (Fig. 4D).

Disc cells cultivated into 3D systems showed a high capability to reacquire their original phenotype based on the detected markers. A weak expression of Sox-9 and Col $2 \alpha 1$ was detected in cell seeded PU-fibrin structures only after 3 days of culture (data not shown), reaching stronger levels at day 14.

\section{Proliferation ability and extracellular matrix protein content in PU-fibrin structures}

Biochemical analysis of DNA content in cell seeded PUfibrin structures confirmed the observations of DAPI staining (Fig. 3). The DNA content constantly increased throughout the culture reaching a maximal value of $3.58 \pm$ $0.79 \mu \mathrm{g}$ DNA per PU-fibrin structure after 14 days (Fig. 5A). Cells proliferated during the first 7 days with a DNA content of $1.71 \pm 0.21 \mu \mathrm{g}$ after 3 days, which then significantly increased (2-fold) within the next 4 days up to $3.27 \pm 0.86 \mu \mathrm{g}$ (Fig. $5 \mathrm{~A}$ ).

The capability of cells to synthesize extracellular matrix proteins was investigated by analyzing the content of collagen and glycosaminoglycans (GAG). The collagen (Fig. 5B) and GAG content (Fig. 5C) per DNA content showed a substantial time-dependent increase over the complete culture time. The retained GAG content within the PU-fibrin structure was measured, whereas content released into medium was not analyzed. The collagen to DNA ratio significantly increased (1.9-fold) after 14 days of culture while remaining constant in the first 7 days of cell adaptation (Fig. 5B). In contrast, GAG to DNA ratio increased significantly over the complete culture time (Fig. $5 \mathrm{C}$ ) reaching a maximum value of 2.5 -fold in 11 days (day 14 of culture minus day 3 days of pre-culture in proliferation differentiation medium).

\section{Discussion}

Considering the current treatment modalities for disc degeneration that will generally lead to a loss of function of the intervertebral disc, a tissue engineered approach represents a promising strategy for the treatment or even prevention of painful, mildly to moderately degenerated discs. However, several issues need to be solved before potential treatment modalities can be turned into clinical realities. The biological environment of normal NP with acidic $\mathrm{pH}$, low oxygen levels and paucity of nutrients make the construction of $3 \mathrm{D}$ functional tissue a very complex process. The current clinical trials investigate new artificial biomaterials (ClinicalTrials.gov Identifier NCT00589797, NCT00215306, NCT00640029), in order to simply substitute the native IVD, while only one is focusing its attention on the IVD regeneration. In particular, its purpose is to demonstrate the effectiveness and safety of a single intradiscal injection of rhGDF-5, a growth and differentiation factor, into a degenerating single spinal disc of patients with early disc degeneration (ClinicalTrials.gov Identifier NCT00813813).

The aim of the present in vitro study was to investigate the potential of a polyurethane-fibrin-based cell-seeded scaffold combination which maintains autologous disc cells after reimplantation.

A method of direct injection of the cell suspension was applied into NPs of rabbits to augment mildly degenerated discs. Even though the procedure seems to be easy to apply, its success was limited by a low survival rate of the implanted cells and leakage of cells through the injection site (Bertram et al., 2005).

To overcome the disadvantages of a simple cellsolution injection, various studies have been performed using cells embedded in gel-like matrices (poly-L lactic acid, gelatin, collagen-hyaluronan, alginate, chitosan, fibrin) (Brown et al., 2005; Roughley et al., 2006; Halloran et al., 2008; Richardson et al., 2006; Kluba et al., 2005; Yang et al., 2008). Although these studies revealed that the correct matrix formation (including aggrecan, collagen type I and II) can be displayed in the matrix material, the hydrogels do not supply the required initial mechanical stability to the implanted structure.

Hence, augmenting a degenerated NP by applying a cell-seeded biomaterial might be a more successful approach. Various materials have been studied in vitro, and cultured with animal or human cells obtained either from the AF or NP (Yang et al., 2005; Revell et al., 2007; Halloran et al., 2008; Kluba et al., 2005; Gruber et al., 2004). However, most of the materials tested so far fulfill only some of the criteria required but fail in others. In summary, fundamental criteria focusing on intervertebral disc regeneration are: (i) physical and mechanical stability for load-bearing applications but also suitable for minimal invasive surgery if not even injectable without cell depletion; (ii) ability to carry cells and allow sufficient cell attachment, proliferation, and migration into the surrounding matrix; (iii) enhancement of extracellular matrix synthesis to allow tissue formation and remodeling, and (iv) biocompatibility (Chan and Leong, 2008). The ultimate goal of such a scaffold is to unify the criteria posing a challenge to be born.

In order to unite the advantages, in the present study a material structure was applied which combines crucial properties for clinical use: injectable, porous, shape-stable, biologically degradable and biocompatible. At the 
beginning of our study, preconditioned PU spheroids were shown to enable cell adhesion and proliferation. However, it is believed that synthesized proteins diffuse out of the highly porous structure and cannot be sufficiently incorporated into the extracellular matrix, resulting in a prolonged limiting effect of the material (Grad et al., 2003). Here, spheroids were combined with fibrin hydrogel and already showed in the first week of culture a migration from PU particles into the fibrin matrix. After two weeks cells reached a homogenous distribution within the composite comparable to cells cultured in pure fibrin. Eyrich et al. (2007b) and Lee et al. (2005) observed a similar material combination for use in cartilage tissue engineering and found it advantageous due to improved cell distribution and extensive development of extracellular matrix.

Similar to previous studies, performed on human and canine disc cells within hydrogel carriers, our results showed the ability of the disc cells to synthesize extracellular matrix into fibrin and thus a biosynthetic behavior comparable to that of cells in native tissue (Gruber et al., 2004; Rong et al., 2002). Moreover, unlike those studies, the fibrin was combined with PU spheroids in order to supply more elasticity, resistance and rigidity to the implants for further clinical applications.

In our experiments, we were able to show increased collagen content as a major component of the extracellular matrix. In addition, GAG content was increased in the scaffold over time, therefore indicating increased capacity for water retention, an important feature of the NP in vivo. However, it is noted that GAG content was not determined in medium supernatant since a substantial amount of GAG might have diffused into the medium, as demonstrated by Lee et al. (2005). The augmentation of the extracellular matrix, a complex and stable network of proteoglycans (predominantly aggrecan) and collagen, plays an important role in withstanding in vivo mechanical loading and is therefore an important variable to be determined for novel biomaterials.

Besides the ability to synthesize collagen and GAG, a more detailed phenotypic characterization of the cells is necessary, especially when the fact that intervertebral disc cells are prone to dedifferentiation during monolayer expansion is taken into consideration. It was previously shown by Kluba et al. (2005) that collagen type II expression is reduced during monolayer culture, a finding that we were able to confirm. However, it is known that disc cell culture in appropriate 3D structures as the pellet system may at least partly revert to their original features (Gan et al., 2003). Thus, gene expression of several chondrocytic and/or disc cell markers were analyzed in disc explants, cells expanded in monolayer as well as cells cultured on the PU-fibrin scaffold and in pellet culture.

Expansion of autologous disc cells is required since the source of cells during discectomy is quite limited. However, we found severe alterations in the gene expression profile after monolayer expansion. While aggrecan expression remained unchanged in all culture systems, substantial changes were found for collagen expression. Collagen type II expression of biopsies was downregulated during monolayer expansion, data obtained being similar to that presented by Kluba et al. (2005). However, we were able to demonstrate clearly that collagen type II expression in 3D cultures, PU-fibrin structure and pellet was upregulated compared to monolayer cultures.

We also analyzed disc cell cultures in respect of their ability to redifferentiate not only by detecting matrixrelated markers but also by NP-specific mRNA expression. Although different markers have been suggested for NP cells in the last few years, no ideal single marker has been identified so far, thus leading to the conclusion that the expression of a set of genes needs to be analyzed, such as HIF-1 (hypoxia induced factor) (Rajpurohit et al., 2002), GLUT-1 (glucose transporter) (Rajpurohit et al., 2002), MMP2 (matrix metalloproteinase 2) (Rajpurohit et al., 2002), CD24 (glycosylphosphat-idylinositol-anchored cell surface protein) (Fujita et al., 2005) and Sox9 (Paul et al., 2003). Both HIF-1 and GLUT-1 are related to the NP metabolic activity. The data presented show expressions of HIF-1, GLUT-1 and CD24 before and after isolation as well as monolayer-expansion when seeded into the PUfibrin material combination, indicating that all the cultures analyzed maintain their phenotypic character.

MMP-2 was also measured and found to increase slightly during 3D cultures, potentially indicating the induction of remodeling processes, characterized by matrix formation of disc cells and degradation of fibrin structure. The reason of MMP2 expression in addition during monolayer culture is not yet known. Even more interesting is the expression of Sox9 in 3D cultures. Paul et al. (2003) studied the potential use of a recombinant adenoviral vector expressing Sox9 in human disc cells among others. It was shown that the expression of the chondrocyte-specific transcription factor clearly stimulated collagen type II synthesis in vitro. These mRNA level data also reflect our findings of increased matrix proteins (total collagen and aggrecan content). Thus, initial expression of Sox9 and collagen type II after 14 days may indicate the synthesis of an appropriate extracellular matrix.

The long-term goal of this study was to develop new regenerative treatment options for degenerated disc augmentation using cell-seeded biomaterial for a minimal invasive reimplantation via an arthroscopic procedure. As a first step, we demonstrated the suitability of a PU-fibrin biomaterial structure as a carrier scaffold for disc cells. In this current setting, disc cells are isolated from biopsies and expanded in monolayer before being seeded into the scaffold and combined with fibrin hydrogel for reimplantation. Therefore, short expansion periods are necessary to avoid severe dedifferentiation and to create a reasonable time for patients between the first surgery (discectomy) and the reimplantation. We were able to show that cells redifferentiate in PU-fibrin composites based on the presented marker design, underlining the fact that it is not the initial number of implanted cells which is decisive (Nomura et al., 2001). Instead, the ability to synthesize and incorporate extracellular matrix protein into the scaffold seems to be more important. 


\section{Conclusion}

The current study provides first evidence that the polymerfibrin structure developed is suitable to augment earlystage degenerated intervertebral disc. The idea at the base of this study was to generate a 3D system able to supply the degenerated nucleus pulposus with autologous fresh cells, in vitro expanded, and so to restore the spine properties. The injectability of the fibrin as well as the resistance of the PU makes of this model a potential candidate for the IVD tissue engineering. Moreover, human disc cells cultivated in the PU-fibrin material, reaching a homogenous 3D distribution in the fibrin, re-acquired their original phenotype and capability to synthesize extracellular matrix molecules. Even though further optimizations are necessary before the translation of this promising approach into a widely applicable treatment modality, our results indicate the potential to lay the base for an advanced, cost-efficient and minimally invasive celltherapeutic approach which takes medical needs into account.

\section{Acknowledgments}

Special thanks go to The Innovation Promotion Agency (CTI) in Switzerland for funding this study. The authors are also indebted to Tobias Wermelinger (Zurich University of Applied Sciences, Waedenswil, Switzerland) for his assistance with the gene expression analysis. Further thanks go to Dr. Karin Wuertz (University Hospital Balgrist, Zurich, Switzerland) for the revision of the manuscript.

\section{References}

Barbosa I, Garcia S, Barbier-Chassefière V, Caruelle JP, Martelly I, Papy-García D (2003) Improved and simple micro assay for sulfated glycosaminoglycans quantification in biological extracts and its use in skin and muscle tissue studies. Glycobiology 13: 647-653.

Bertram H, Kroeber M, Wang H, Unglaub F, Guehring T, Carstens C, Richter W (2005) Matrix-assisted cell transfer for intervertebral disc cell therapy. Biochem Biophys Res Commun 331: 1185-1192.

Brown RQ, Mount A, Burg KJ (2005) Evaluation of polymer scaffolds to be used in a composite injectable system for intervertebral disc tissue engineering. J Biomed Mater Res A 74: 32-39.

Caterson EJ, Nesti LJ, Li WJ, Danielson KG, Albert TJ, Vaccaro AR, Tuan RS (2001) Three-dimensional cartilage formation by bone marrow-derived cells seeded in polylactide/alginate amalgam. J Biomed Mater Res 57: 394-403.

Chan BP, Leong KW (2008) Scaffolding in tissue engineering: general approaches and tissue-specific consideration. Eur Spine J 17: 467-479.

Di Martino A, Vaccaro AR, Lee JY, Denaro V, Lim MR (2005) Nucleus pulposus replacement: basic science and indications for clinical use. Spine 30: S16-S22.
Eyrich D, Brandl F, Appel B, Wiese H, Maier G, Wenzel M, Staudenmaier R, Goepferich A, Blunk T (2007a) Longterm stable fibrin gels for cartilage engineering. Biomaterials 28: 55-65.

Eyrich D, Wiese H, Maier G, Skodacek D, Appel B, Sarhan H, Tessmar J, Staudenmaier R, Wenzel MM, Goepferich A, Blunk T (2007b) In vitro and in vivo cartilage engineering using a combination of chondrocyte-seeded long-term stable fibrin gels and polycaprolactone-based polyurethane scaffolds. Tissue Eng 13: 2207-2218.

Fujita N, Miyamoto T, Imai J, Hosogane N, Suzuki T, Yagi M, Morita K, Ninomiya K, Miyamoto K, Takaishi H, Matsumoto M, Morioka H, Yabe H, Chiba K, Watanabe S, Toyama Y, Suda T (2005) CD24 is expressed specifically in the nucleus pulposus of intervertebral discs. Biochem Biophys Res Commun 338: 1890-1896.

Gan JC, Ducheyne P, Vresilovic EJ, Swaim W, Shapiro IM (2003) Intervertebral disc tissue engineering I: Characterization of the nucleus pulposus. Clin Orthop Relat Res 411: 305-314.

Gao W, Gao Y, Zhang G, Song L, Sun B, Shi J (2005) Hypoxia-induced expression of HIF-1alpha and its target genes in umbilical venous endothelial cells of Tibetans and immigrant Han. Comp Biochem Physiol C Toxicol Pharmacol 141: 93-100.

Gogolewski S, Gorna K, Turner AS (2006) Regeneration of bicortical defects in the iliac crest of estrogen-deficient sheep, using new biodegradable polyurethane bone graft substitutes. J Biomed Mater Res A 77: 802-810.

Gogolewski S, Gorna K (2007) Biodegradable polyurethane cancellous bone graft substitutes in the treatment of iliac crest defects. J. Biomed Mater Res A 80: 94-101.

Grad S, Kupcsik L, Gorna K, Gogolewski S, Alini M (2003) The use of biodegradable polyurethane scaffolds for cartilage tissue engineering: potential and limitations. Biomaterials 24: 5163-5171.

Grad S, Lee CR, Wimmer MA, Alini M (2006) Chondrocyte gene expression under applied surface motion. Biorheology 43: 259-269.

Gruber HE, Leslie K, Ingram J, Norton HJ, Hanley EN (2004) Cell-based tissue engineering for the intervertebral disc: in vitro studies of human disc cell gene expression and matrix production within selected cell carriers. Spine J 4: 44-55.

Guelcher SA, Patel V, Gallagher KM, Connolly S, Didier JE, Doctor JS, Hollinger JO (2006) Synthesis and in vitro biocompatibility of injectable polyurethane foam scaffolds. Tissue Eng 12: 1247-1259.

Halloran DO, Grad S, Stoddart M, Dockery P, Alini M, Pandit AS (2008) An injectable cross-linked scaffold for nucleus pulposus regeneration. Biomaterials 29: 438447.

Hoemann CD, Sun J, Chrzanowski V, Buschmann MD (2002) A multivalent assay to detect glycosaminoglycan, protein, collagen, RNA, and DNA content in milligram samples of cartilage or hydrogel-based repair cartilage. Anal Biochem 300: 1-10.

Kluba T, Niemeyer T, Gaissmaier C, Gründer T (2005) Human anulus fibrosus and nucleus pulposus cells of the 
intervertebral disc: effect of degeneration and culture system on cell phenotype. Spine 30: 2743-2748.

Lee CR, Grad S, Gorna K, Gogolewski S, Goessl A, Alini M (2005) Fibrin-polyurethane composites for articular cartilage tissue engineering: a preliminary analysis. Tissue Eng 11: 1562-1573.

Lin Y, Luo E, Chen X, Liu L, Qiao J, Yan Z, Li Z, Tang W, Zheng X, Tian W (2005) Molecular and cellular characterization during chondrogenic differentiation of adipose tissue-derived stromal cells in vitro and cartilage formation in vivo. J Cell Mol Med 9: 929-939.

Liu Y, Webb K, Kirker KR, Bernshaw NJ, Tresco PA, Gray SD, Prestwich GD (2004) Composite articular cartilage engineered on a chondrocyte-seeded aliphatic polyurethane sponge. Tissue Eng 10: 1084-1092.

Mayer HM (2005) Total lumbar disc replacement. J Bone Joint Surg Br 87: 1029-1037.

Nomura T, Mochida J, Okuma M, Nishimura K, Sakabe K (2001) Nucleus pulposus allograft retards intervertebral disc degeneration. Clin Orthop Relat Res 389: 94-101.

Paesold G, Nerlich AG, Boos N (2007) Biological treatment strategies for disc degeneration: potentials and shortcomings. Eur Spine J 16: 447-468.

Paul R, Haydon RC, Cheng H, Ishikawa A, Nenadovich N, Jiang W, Zhou L, Breyer B, Feng T, Gupta P, He TC, Phillips FM (2003) Potential use of Sox9 gene therapy for intervertebral degenerative disc disease. Spine 28: 755 763.

Peretti GM, Randolph MA, Villa MT, Buragas MS, Yaremchuk MJ (2000) Cell-based tissue-engineered allogeneic implant for cartilage repair. Tissue Eng 6: 567 576.

Rajpurohit R, Risbud MV, Ducheyne P, Vresilovic EJ, Shapiro IM (2002) Phenotypic characteristics of the nucleus pulposus: expression of hypoxia inducing Factor1, Glucose Transporter-1 and MMP-2. Cell Tissue Res 308: 401-407.

Revell PA, Damien E, Di Silvio L, Gurav N, Longinotti C, Ambrosio L (2007) Tissue engineered intervertebral disc repair in the pig using injectable polymers. J Mater Sci Mater Med 18: 303-308.

Richardson SM, Curran JM, Chen R, Vaughan-Thomas A, Hunt JA, Freemont AJ, Hoyland JA (2006) The differentiation of bone marrow mesenchymal stem cells into chondrocyte-like cells on poly-L-lactic acid (PLLA) scaffolds. Biomaterials 27: 4069-4078.

Robey IF, Lien AD, Welsh SJ, Baggett BK, Gillies RJ (2005) Hypoxia-inducible factor-1 alpha and the glycolytic phenotype in tumors. Neoplasia 7: 324-330.

Rong Y, Sugumaran G, Silbert JE, Spector M (2002) Proteoglycans synthesized by canine intervertebral disc cells grown in a type I collagen-glycosaminoglycan matrix. Tissue Eng 8: 1037-1047.

Roughley P, Hoemann C, DesRosiers E, Mwale F, Antoniou J, Alini M (2006) The potential of chitosan-based gels containing intervertebral disc cells for nucleus pulposus supplementation. Biomaterials 27: 388-396.

Sanchez C, Deberg MA, Piccardi N, Msika P, Reginster JY, Henrotin YE (2005) Subchondral bone osteoblasts induce phenotypic changes in human osteoarthritic chondrocytes. Osteoarthritis Cartilage 13: 988-997.

Séguin CA, Grynpas MD, Pilliar RM, Waldman SD, Kandel RA (2004) Tissue engineered nucleus pulposus tissue formed on a porous calcium polyphosphate substrate. Spine 29: 1299-1306.

Shan B, Zhuo Y, Chin D, Morris CA, Morris GF, Lasky JA (2005) Cyclin-dependent kinase 9 is required for Tumor Necrosis Factor-alpha-stimulated Matrix Metalloproteinase-9 expression in human lung adenocarcinoma cells. J Biol Chem 280: 1103-1111.

Urban JP, Roberts S (2003) Degeneration of the intervertebral disc. Arthritis Res Ther 5: 120-130.

Wiese H, Maier G. (2006) Open-pored polyurethane foam without skin formation, formulation for the production thereof and use thereof as a carrier material for cell and tissue cultures or medicaments. Patent (WO 2006032501 A1).

Yang L, Kandel RA, Chang G, Santerre JP (2009) Polar surface chemistry of nanofibrosus polyurethane scaffold affects annulus fibrosus cell attachment and early matrix accumulation. J Biomed Mater Res A, in press.

Yang SH, Chen PQ, Chen YF, Lin FH (2005) Gelatin/ chondroitin-6-sulfate copolymer scaffold for culturing human nucleus pulposus cells in vitro with production of extracellular matrix. J Biomed Mater Res B Appl Biomater. 74: 488-494.

Yang SH, Wu CC, Shih TT, Chen PQ, Lin FH (2008) Three-dimensional culture of human nucleus pulposus cells in fibrin clot: comparisons on cellular proliferation and matrix synthesis with cells in alginate. Artif Organs 32: 70-73.

\section{Discussion with Reviewers}

Reviewer I: It is known that fibrin is degraded by proteolytic enzymes that may be released by the cells. Thus it can be assumed that the fibrin structure might be weakened after 14 days of culture in medium without proteinase inhibitor. Was any degradation of the fibrin component observed after 14 days of culture?

Authors: The cell seeded PU-fibrin composites, after two weeks of culture, did not show any morphological difference compared to non-seeded structures (blank samples). Moreover, composites cultured for 3 and 7 days also showed a stable structure. Certainly, for a longer period of culture, the fibrin is degraded but at the same time it would be replaced with extracellular matrix components, which as shown in the present study are synthesized in the first weeks of culture.

Reviewer I: Was the capability of proliferation and matrix synthesis (DNA, GAG, collagen content) different in cells from donors $<40$ years versus donors $>40$ years of age?

Reviewer III: How did the age of the patient and the degree of degeneration of the IVD influence the outcome of the cell culture?

Authors: With age, similar as in cases of degeneration, the IVD undergoes a variety of changes with a lower 
synthesis and accumulation of the extracellular matrix proteins as collagen and aggrecan, and increasing levels of degrading enzymes. Intervertebral disc cells isolated from younger donors proliferated faster versus the older ones. Regarding cell capability to synthesize extracellular matrix proteins, it is, however, necessary to mention that the IVD degeneration level as well as the health state of the patient influences the disc-cell metabolism. Because of the high variability of the condition of the donors a final conclusion on the age effects on cell biosynthesis cannot be drawn. However, we could hypothesize that better results can be obtained with the use of cells isolated from young/healthy discs. A recent in vitro study, performed on disc cells isolated from bovine caudal spines, has demonstrated that mature cells are not able to synthesize extracellular matrix at the same level as younger cells (Kandel et al., 2007).

Reviewer I: Can the authors comment on the effect of the chondrogenic supplements TGF- $\beta$ and dexamethasone on gene expression and matrix synthesis? Was redifferentiation of disc cells in the $3 \mathrm{D}$ environment also investigated in control cultures without chondrogenic supplements?

Authors: All culture experiments were performed with TGF- $\beta$ and dexamethasone. As known in the literature, TGF- $\beta$ and dexamethasone, acting as anabolic regulators of disc cell metabolism, are able to increase and maintain the cell chondrocyte-like phenotype. Because of this knowledge and of our know-how on the maintenance of the chondrogenic metabolism of disc cells in pellet cultures, we decided to enrich the culture medium with the supplements mentioned. Our final aim was to optimize the in vitro experimental conditions and compare our models to an established positive control (pellet system).

Reviewer III: How would the application of pressure to the cell culture influence the maturation of matrix and cells?

Authors: Under physiological conditions, the IVD is daily exposed to different and complex physical stimuli during mechanical loads such as compressive and shear stresses, osmotic and hydrostatic pressure. Intense work, in vitro and in vivo, demonstrated that disc cells responses depend on pressure magnitude, frequency as well as on the loading type. High magnitude or frequency of loading induce catabolic cell responses with down-regulation of matrix forming and up-regulation of matrix degrading enzymes, cell death and degeneration; while low mechanical applications give rise to a stimulatory effect on biosynthesis, improving the expression of collagen and proteoglycans.

\section{Additional Reference}

Kandel RA, Hamilton D, Séguin C, Li SQ, Arana C, Pilliar R (2007) An in vitro tissue model to study the effect of age on nucleus pulposus cells. Eur Spine J 16: 21662173. 